\title{
Effect of thymoquinone on Fusobacterium nucleatum-associated biofilm and inflammation
}

\author{
AYANO TADA $^{1}$, HARUYUKI NAKAYAMA-IMAOHJI ${ }^{1}$, HISASHI YAMASAKI $^{2}$, MIAD ELAHI $^{1}$, \\ TAMIKO NAGAO ${ }^{3}$, HIROFUMI YAGI ${ }^{1}$, MASAO ISHIKAWA ${ }^{4,5}$, KOJI SHIBUYA ${ }^{4}$ and TOMOMI KUWAHARA ${ }^{1}$ \\ ${ }^{1}$ Department of Microbiology, Faculty of Medicine, Kagawa University, Takamatsu, Kagawa 761-0793; \\ ${ }^{2}$ Division of Biology, Hyogo College of Medicine, Nishinomiya, Hyogo 663-8501; ${ }^{3}$ Faculty of Nursing, \\ Shikoku University, Ohjin, Tokushima 771-1192; ${ }^{4}$ Laboratory for Oral Health Science, Tokyo 103-0012; \\ ${ }^{5}$ Department of Translational Research, Tsurumi University School of Dental Medicine, Yokohama 230-8501, Japan
}

Received November 7, 2019; Accepted January 29, 2020

DOI: $10.3892 / \mathrm{mmr} .2020 .11136$

\begin{abstract}
Periodontitis affects oral tissues and induces systemic inflammation, which increases the risk of cardiovascular disease and metabolic syndrome. Subgingival plaque accumulation is a trigger of periodontitis. Fusobacterium nucleatum (FN) contributes to subgingival biofilm complexity by intercalating with early and late bacterial colonizers on tooth surfaces. In addition, inflammatory responses to $\mathrm{FN}$ are associated with the progression of periodontitis. Nigella sativa Lin. seed, which is known as black cumin (BC), has been used as a herbal medicine to treat ailments such as asthma and infectious diseases. The current study examined the inhibitory effect of BC oil and its active constituents, thymol (TM) and thymoquinone (TQ), on FN-associated biofilm and inflammation. FN-containing biofilms were prepared by co-cultivation with an early dental colonizer, Actinomyces naeslundii (AN). The stability and biomass of FN/AN dual species biofilms were significantly higher compared with FN alone. This effect was retained even with prefixed cells, indicating that FN/AN co-aggregation is mediated by physicochemical interactions with cell surface molecules. FN/AN biofilm formation was significantly inhibited by $0.1 \%$ TM or TQ. Confocal laser scanning microscopy indicated that treatment of preformed FN/AN biofilm with $0.01 \%$ of BC, TM or TQ significantly reduced biofilm thickness, and TQ demonstrated a cleansing effect equivalent to that of isopropyl methylphenol. TQ dose-dependently suppressed TNF- $\alpha$ production from a human monocytic cell line, THP-1 exposed to FN, yet showed no toxicity to THP-1 cells. These results indicated that oral
\end{abstract}

Correspondence to: Professor Tomomi Kuwahara, Department of Microbiology, Faculty of Medicine, Kagawa University, 1750-1 Miki, Takamatsu, Kagawa 761-0793, Japan

E-mail: tomomi@med.kagawa-u.ac.jp

Key words: Fusobacterium, dental plaque, biofilm, black cumin, thymoquinone, cleansing, oral care, periodontitis hygiene care using TQ could reduce $\mathrm{FN}$-associated biofilm and inflammation in gingival tissue.

\section{Introduction}

The human oral cavity is estimated to harbor nearly 700 bacterial species (1). Some oral bacteria form biofilms called dental plaque on tooth surfaces. Dental plaque accumulation is responsible for dental caries or periodontal diseases. Oral biofilm formation is initiated by early colonizing bacteria such as Streptococcus and Actinomyces that adhere to the pellicle layer on tooth surfaces (2).

Fusobacterium nucleatum (FN) is a Gram-negative oral anaerobe that plays an important role in subgingival plaque maturation by bridging early and late colonizers $(2,3)$. FN colonizes the oral cavity of patients with periodontal diseases but is also found in healthy subjects (4). Since the number of FN increases as periodontal disease progresses, this anaerobe is recognized as a periodontal pathogen (4). Inflammation evoked by lipopolysaccharide of FN is reported to result in chronic periodontitis and alveolar bone absorption (5-7). In addition, FN produces methyl mercaptan and hydrogen sulfate that are responsible for oral malodor $(8,9)$. The FadA adhesion protein of FN was recently shown to activate Wnt/ $\beta$-catenin signaling by binding to E-cadherin, which induces colon epithelial proliferation and colon cancer (10). Thus, FN has attracted attention as an etiologic agent not only for periodontal diseases but also for other systemic diseases (11).

Chronic inflammation due to periodontitis is implicated in various systemic conditions such as those associated with diabetes mellitus, cardiovascular diseases, pulmonary diseases, obesity, low-weight birth and preterm birth (12). Oral hygiene is an effective prophylactic measure for various systemic diseases. Accordingly, antimicrobial chemicals such as cetylpyridinium chloride (CPC), chlorhexidine gluconate and isopropyl methylphenol are used as additives in commercially available oral hygiene care products such as toothpaste and mouth rinse solution. However, some of these chemicals are associated with cytotoxicity or side effects $(13,14)$.

Natural products such as herbal plant extracts can be used as alternative oral care reagents that have fewer adverse 
effects (15). Essential oil from Matricaria chamomilla is known to possess antimicrobial activity and oral rinses containing this oil reduced oropharynx colonization by Staphylococcus aureus and Streptococcus pneumoniae in intensive care unit patients (16). Another study reported that the medicinal plant, Baccharis dracunculifolia, reduced dental plaque in healthy individuals (17), and a combination of herbal essential oils promoted chlorhexidine gluconate-mediated inhibition of biofilm formation by Streptococcus mutans or Lactobacillus plantarum (18).

Nigella sativa L. (Ranunculaceae), commonly known as black cumin (BC), is an annual flowering plant native to South and Southeast Asia. N. sativa has a long history as a medicinal plant for treatment of various ailments such as metabolic syndrome as well as gastrointestinal, neuronal, respiratory, urinary, reproductive disorders (19). BC seeds have been shown to possess anti-diabetic, anti-cancer, anti-inflammatory, immunomodulatory, antioxidant, antimicrobial, analgesic, spasmolytic, bronchodilatory and hepatoprotective properties $(19,20)$. Most biological activities of BC are related to its essential oil components. Thymoquinone (TQ) is a major (30-48\%) component of BC essential oil and contributes to its therapeutic effects $(19,21)$. In a rat model of periodontitis, Ozdemir et al (22) showed that gastric feeding of TQ (10 mg/kg, daily for 11 days) significantly reduced gingival inflammation and alveolar bone loss. Based on these findings, TQ is expected to have anti-bacterial and anti-inflammatory properties that could prevent periodontal diseases. However, the effect of TQ on FN-associated biofilms remains to be elucidated.

In this study, we evaluated the inhibitory effect of TQ on $\mathrm{FN}$-associated biofilm formation and discuss the therapeutic potential of TQ for periodontal disease.

\section{Materials and methods}

Bacterial stains and culture conditions. The bacterial strains used in this study were Fusobacterium nucleatum ATCC25586 (FN), Actinomyces naeslundii X600 (AN) and Streptococcus mitis ATCC 903 (SM). These bacterial strains were cultured anaerobically in BHIS broth using the AnaeroPack System (Mitsubishi Gas Chemical Company, Inc.). BHIS broth is Brain Heart Infusion medium (Eiken Chemical Co.) supplemented with $5 \mu \mathrm{g} / \mathrm{ml}$ hemin (Sigma-Aldrich), $0.1 \%$ L-cysteine hydrochloride monohydrate (Sigma-Aldrich), $0.5 \%$ yeast extract (Becton Dickinson and Company) and $0.375 \%$ D-glucose (Nacalai Tesque).

Preparation of biofilm containing $F N$. Frozen stocks $\left(-80^{\circ} \mathrm{C}\right)$ of FN, AN or SM were streaked onto BHIS agar plates and incubated anaerobically for 48-72 h. Single colonies of each strain were used to inoculate $3 \mathrm{ml}$ of BHIS broth that was then incubated at $37^{\circ} \mathrm{C}$ for $24 \mathrm{~h}$ in an anaerobic chamber conditioned with $80 \% \mathrm{~N}_{2}, 10 \% \mathrm{CO}_{2}, 10 \% \mathrm{H}_{2}$. After the incubation, the optical density of each culture at $590 \mathrm{~nm}\left(\mathrm{OD}_{590}\right)$ was adjusted to 0.1 with BHIS broth. Subsequently, FN culture was mixed with equal amounts of cultured $\mathrm{AN}$ and/or SM, and $1 \mathrm{ml}$ of the culture mixture was dispensed into 24-well polystyrene tissue culture plates. Gram-staining was performed with the culture used for biofilm preparation to exclude contamination by other bacteria. Biofilms formed by $4 \%$ paraformaldehyde (PFA)-fixed
FN and/or AN were also examined. For fixation, FN and/or AN cells collected from overnight culture were suspended in $4 \%$ PFA in phosphate-buffered saline (PBS, pH 7.4) and incubated $30 \mathrm{~min}$ at room temperature. After the fixed cells were washed with PBS three times, the cells were resuspended in BHIS to 0.1 $\mathrm{OD}_{590}$ and transferred to multi-titer wells. Dual species biofilms was prepared on the bottom of wells in 24-well plates following anaerobic incubation at $37^{\circ} \mathrm{C}$ for $24 \mathrm{~h}$. To prepare biofilms containing $\mathrm{FN}$ alone, $1 \mathrm{ml}$ of $\mathrm{FN}$ culture $\left(\mathrm{OD}_{590}\right.$ adjusted to 0.1) was applied to the wells and incubated as described above. Scanning electron microscopy was used to assess the prevalence of FN in biofilms. To prepare the biofilms, sterilized plastic discs (Sensi-Disc, $13.5 \mathrm{~mm}$, Sumitomo Bakelite, Co., Ltd.) were inserted into wells before applying bacterial mixtures. Biofilms that formed on the discs were fixed with $2 \%$ glutaraldehyde in $0.1 \mathrm{M}$ cacodylate buffer ( $\mathrm{pH}$ 7.2). After fixation, the biofilms were dehydrated in a graded ethanol series and dried in a Hitachi PCP-2 critical point drying apparatus. The discs were coated with platinum/palladium in a Hitachi E-102 sputter coater and examined with a JEOL JCM-6000 scanning electron microscope.

Chemicals. Test reagents used in this study were thymol (TM, $>98 \%$ ), TQ (>99\%), isopropyl methylphenol (IPMP, >99\%) and CPC (>99\%). TQ, IPMP and CPC were purchased from Sigma-Aldrich Co.). TM was obtained from Kanto Chemical Co., Inc. BC was prepared from $500 \mathrm{~g}$ of BC seed as described previously (23), and $3.6 \mathrm{~g}$ of the oil was obtained (recovery rate, $0.72 \%$ ).

Inhibitory effect of TM or TQ on FN-containing biofilm formation. Equal volumes of $\mathrm{FN}$ and $\mathrm{AN}$ cultures adjusted to $\mathrm{OD}_{590} 0.1$ were mixed and dispensed into 24-well polystyrene tissue culture plates. Before incubation, TM or TQ was added to the culture at a final concentration of $0.1 \%$. After anaerobic incubation at $37^{\circ} \mathrm{C}$ for $24 \mathrm{~h}$, the amount of biofilm mass formed on the bottom of the wells was assessed using crystal violet (Wako Pure Chemical) staining. After removing the test media, the biofilms were gently washed three times with $1 \mathrm{ml}$ saline and then stained with $0.4 \mathrm{ml}$ crystal violet solution $(0.01 \%)$ at room temperature for $1 \mathrm{~h}$. Excess crystal violet solution was removed and the stained biofilm was washed with $1 \mathrm{ml}$ saline before eluting the remaining dye with $1 \mathrm{ml}$ acetic acid (33\%, Wako Pure Chemical) and gentle agitation for $30 \mathrm{~min}$ at room temperature. The eluent was transferred to a 96-well plate for measurement at $\mathrm{OD}_{550}$ or $\mathrm{OD}_{600}$ to compare the biofilm mass.

Effect of TQ pretreatment on FN/AN biofilm formation. After anaerobic culture of $\mathrm{FN}$ and $\mathrm{AN}$ in BHIS at $37^{\circ} \mathrm{C}$ overnight, the cells were collected by centrifugation $(6,000 \mathrm{~g}, 5 \mathrm{~min}$, $4 \mathrm{C}$ ), suspended in PBS ( $\mathrm{pH} 7.4$ ) containing $0.05 \%$ TQ and incubated for $30 \mathrm{~min}$ at room temperature in the dark. The cells were again collected by centrifugation and washed three times with PBS before resuspension in BHIS to $\mathrm{OD}_{590}$ to 0.1. FN/AN biofilms were prepared using prefixed and unfixed cell combinations. Biofilm mass was compared by crystal violet staining as described above.

Cleansing effect on FN-containing biofilms. Mixed FN and AN biofilms were washed by gentle agitation with $0.5 \mathrm{ml}$ 
0.01 or $0.05 \% \mathrm{BC}, \mathrm{TM}, \mathrm{TQ}, \mathrm{IPMP}$ or $\mathrm{CPC}$ at $37^{\circ} \mathrm{C}$ for $30 \mathrm{~min}$. After removing the test reagents, crystal violet staining was performed to compare the biofilm mass that remained after treatment as described above. Confocal laser scanning microscopy (CLSM) was also used to evaluate biofilm thickness and bacterial viability after treatment. The remaining biofilm was stained using a LIVE/DEAD ${ }^{\circledR}$ Biofilm Viability kit (Thermo Fisher Scientific, Inc.) according to the manufacturer's instructions. Stained biofilms were observed by CLSM (model LSM700; Carl Zeiss) at 400x magnification and a $488 \mathrm{~nm}$ emission wavelength (15\% laser power). Signal/noise ratio, digital offset and digital gain settings were optimized using untreated biofilm and biofilm treated with $70 \%$ ethanol. The thickness of the remaining biofilm was calculated using ZEN imaging software (ZEN 2012). The live/dead ratio of bacteria in the biofilm was estimated from output data for the arithmetic average luminance using ZEN imaging software.

Anti-inflammatory effect of $T M$ and $T Q$. The human monocytic leukemia cell line THP-1 (JCRB0112.1) was cultured in RPMI1640 medium (Wako Pure Chemical) containing 10\% fetal bovine serum, $100 \mathrm{U} / \mathrm{ml}$ penicillin and $100 \mu \mathrm{g} / \mathrm{ml}$ streptomycin (Pen Strep; Gibco; Thermo Fisher Scientific, Inc.) at $37^{\circ} \mathrm{C}$ and $5 \% \mathrm{CO}_{2}$. After seeding in 24-well plates at an initial cell density of $5 \times 10^{5}$ cells/well, the THP-1 cells were treated with a 5-50 $\mu \mathrm{M} \mathrm{TM}$ or TQ for $1 \mathrm{~h}$ at $37^{\circ} \mathrm{C}$ under $5 \% \mathrm{CO}_{2}$. Subsequently, FN suspension $\left(\mathrm{OD}_{590}=1.0\right)$ in PBS was added to the media at $10 \%(\mathrm{v} / \mathrm{v})$ and incubated for $3 \mathrm{~h}$. The culture supernatants were collected by centrifugation at $600 \mathrm{x} \mathrm{g}$ for 2 min at $4^{\circ} \mathrm{C}$. TNF- $\alpha$ secreted from THP- 1 cells after stimulation by FN was quantified with a Human TNF- $\alpha$ ELISA Ready Set-Go ${ }^{\circledR}$ assay (Thermo Fisher Scientific, Inc.).

Toxicity of TM and TQ toward THP-1 cells. THP-1 cells were treated with TM and TQ as described above. Cell viability was assessed by adding $8 \mu \mathrm{g} / \mathrm{ml} \mathrm{3-(4,5-dimethylthi-}$ azol-2-yl)-2,5-diphenyltetrazolium bromide (MTT; Dojindo Laboratories) to the media. Zinc dibutyldithiocarbamate (ZDBC, $1 \mu \mathrm{g} / \mathrm{ml}$, Wako Pure Chemical Corporation) was used as a positive control.

Statistical analysis. Data are expressed as mean \pm standard deviation. Statistical analyses were performed with StatFlex ver. 6.0 (Artech Co., Ltd.) wherein analysis of variance (ANOVA) was used to compare the means of all groups, followed by Tukey's test (for all combinations) or Dunnett's test (for comparison to control). Statistically significant differences of the live/dead cell ratio in biofilms were assessed by Chi-square test. $\mathrm{P}<0.05$ was considered to indicate a statistically significant difference.

\section{Results}

Preparation of $F N$-containing biofilm. To examine the effect of $\mathrm{BC}$ and its components on $\mathrm{FN}$-containing biofilm, we first prepared single species biofilms containing only FN in microtiter wells. Although FN grew well, it formed thin and fragile biofilms (Fig. 1A). To increase the biofilm stability, FN was co-cultivated with early colonizers in dental plaque, SM and/or AN. Co-cultivation of FN with AN resulted in thick and firm biofilms whereas SM and FN co-cultivation inhibited biofilm formation as revealed by crystal violet staining (Fig. 1A and B). Gram-staining of the dual species biofilm showed co-agglutination of FN (Gram-negative rods) and AN (Gram-positive rods) (Fig. 1C). Scanning electron microscopy also showed that this dual species biofilm contained substantial amounts of needle-shaped FN cells (Fig. 1D). These dual species FN/AN biofilms were used in subsequent experiments.

Inhibitory effect of BC components on $F N$-containing biofilm. We examined whether the BC components TM and TQ inhibited $\mathrm{FN}$-associated biofilm formation. Upon addition of either $0.1 \% \mathrm{TM}$ or TQ, dual species biofilm formation of FN and AN was significantly inhibited as indicated by crystal violet staining that showed decreased biofilm mass (Fig. 2). This effect was likely due to growth inhibition since FN and AN co-cultures were only slightly turbid after anaerobic incubation for $24 \mathrm{~h}$ in the presence of $0.1 \% \mathrm{TM}$ or TQ (data not shown).

Effect of TQ pretreatment on FN/AN biofilm formation. Biofilms were prepared with untreated or TQ-treated FN and AN (Fig. 3). FN pretreated with $0.01 \%$ TQ showed significantly decreased biofilm formation whereas AN pretreated with TQ formed biofilms with efficiencies similar to those seen for untreated AN. For dual species biofilms, biofilm mass was significantly reduced when FN was pretreated with $\mathrm{TQ}$. These results indicate that $\mathrm{FN}$ is more sensitive to TQ than AN.

Cleansing effect of BC components on FN-containing biofilm. After co-cultivation of FN and AN, the dual species biofilms were washed with $0.01 \%$ or $0.05 \% \mathrm{BC}$, TM, TQ, IPMP or CPC. Ethyl alcohol (0.05\%) was also tested as an antimicrobial agent. IPMP, BC, and TQ all significantly reduced biofilm mass (Fig. 4). CLSM analysis was also conducted to examine the biofilm structure after treatment with the test reagents (Fig. 5). Nearly $80 \%$ of bacteria were viable in untreated biofilms, but upon treatment with TQ, IPMP and CPC the percentage of dead cells in the biofilms significantly increased (Fig. 5A and B). Among these agents, CPC had the highest bactericidal activity with over $70 \%$ of bacteria inactivated in the biofilm. All of the test reagents reduced the biofilm thickness (Fig. 5C). Of these, TQ and IPMP were the most effective for dislodging biofilms and decreased the average biofilm thickness to nearly half that achieved with saline.

Effect of TQ on surface interaction between $F N$ and $A N$. We performed a biofilm assay using prefixed $\mathrm{FN}$ and $\mathrm{AN}$ cells to evaluate the effect of TQ on surface interactions between FN and AN. Interestingly, co-incubation of FN and AN increased cell attachment to microtiter wells even after fixation with $4 \%$ PFA (Fig. 6). Washing with $0.01 \%$ TQ, but not TM, significantly reduced the number of attached cells compared to that seen with saline.

Effect of TM and TQ on proinflammaotry response of THP-1 cells to $F N$. We examined the anti-inflammatory activity of TM and TQ in the human monocytic cell line THP-1. Since $\mathrm{FN}$ is well known to have proinflammatory potential, we used FN sonicate as immune sensitizer to assess anti-inflammatory effect of TM or TQ. Before stimulation with FN, THP-1 

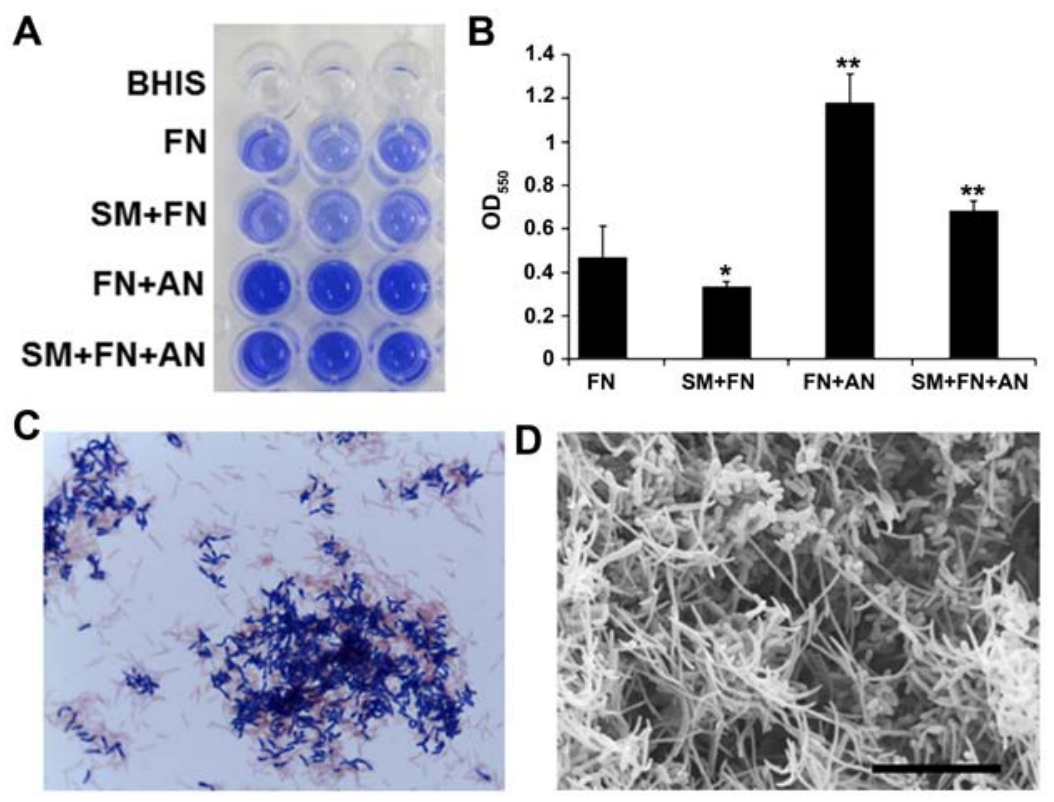

Figure 1. Preparation of FN-containing biofilm. (A) Crystal violet staining assay of single or multi-species biofilm. Triplicate wells are presented. (B) $\mathrm{OD}_{550}$ measurement of bacterial elution from wells shown in Panel A. Data are expressed as mean \pm standard deviation from three repeats. Differences were analyzed using ANOVA followed by Dunnett's test. "P<0.05 or * P $<0.01$. (C) Gram-staining of biofilm scraped from the wells. (D) Scanning electron microscopy of dual species biofilms formed by FN and AN. Scale bar, $10 \mu \mathrm{m}$. FN, Fusobacterium nucleatum; SM, Streptococcus mitis; AN, Actinomyces naeslundii.

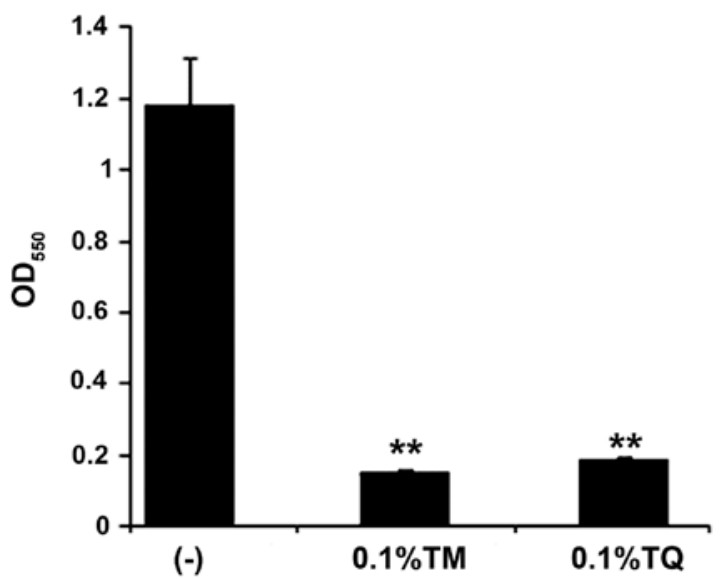

Figure 2. Inhibitory effect of TM and TQ on dual species biofilm formation by FN and AN. Data are expressed as mean \pm standard deviation from three repeats. Differences were analyzed by ANOVA followed by Dunnett's test. ${ }^{* *} \mathrm{P}<0.01$ vs. non-treated sample indicated by (-). TM, thymol; TQ, thymoquinone; FN, Fusobacterium nucleatum; AN, Actinomyces naeslundii.

cells were treated with 5-50 $\mu \mathrm{M}$ TM or TQ. The pretreated THP-1 cells were then exposed to FN, and the amount of TNF- $\alpha$ secreted from the cells was quantified by ELISA. TM or TQ itself did not induce TNF- $\alpha$ production (data not shown). Pretreatment with TM or TQ significantly decreased the amount of TNF- $\alpha$ secretion from THP-1 cells in response to FN (Fig. 7A). However, the anti-inflammatory activity of TQ was higher than that of TM, and the inhibitory effect on TNF- $\alpha$ secretion by TQ was dose-dependent. Pretreatment of cells with $50 \mu \mathrm{M}$ TQ prior to FN exposure decreased TNF- $\alpha$ levels to one-eighth that seen for untreated cells.

We performed a MTT assay to determine whether the inhibitory effects of TM and TQ on TNF- $\alpha$ secretion were cytotoxic effects on THP-1 cells. TM and TQ did not affect

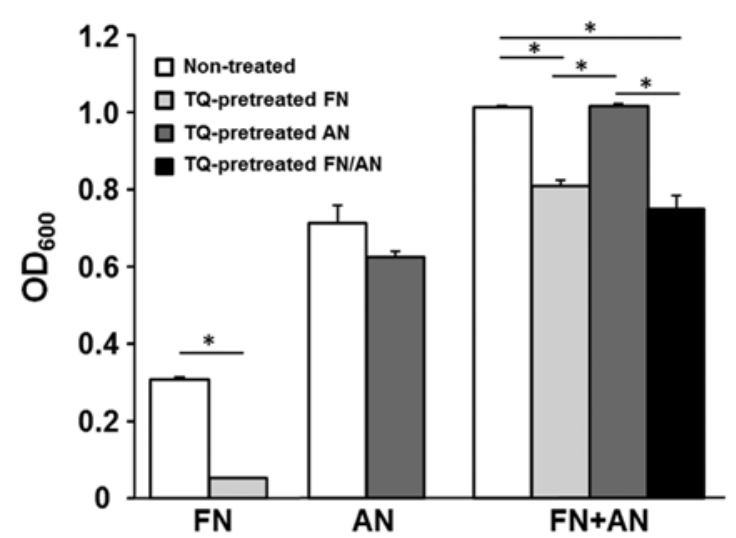

Figure 3. Effect of TQ pretreatment on biofilm formation by FN and/or AN. After FN and/or AN were pretreated with $0.01 \% \mathrm{TQ}$, biofilm formation was compared with the non-treated counterparts. ${ }^{*} \mathrm{P}<0.01$ vs. samples indicated by horizontal lines. TQ, thymoquinone; FN, Fusobacterium nucleatum; AN, Actinomyces naeslundii.

THP-1 cell viability whereas the cytotoxic compound ZDBC did show a toxic effect, as did $50 \mu \mathrm{M} \mathrm{CPC} \mathrm{(Fig.} \mathrm{7B).}$

\section{Discussion}

Although apparent virulence factors such as exotoxins have not yet been identified in FN, the anaerobe is considered to be a periodontal pathogen based on its crucial role in the development of dental plaque that involves bridging diverse types of oral bacteria during biofilm formation (3). The abundance of $\mathrm{FN}$ in the oral microbiome is also known to increase in individuals with periodontitis $(24,25)$. Eradication of FN, which is expected to reduce subgingival biofilm mass and inflammatory responses to this anaerobe, is one therapeutic strategy for periodontitis. Compounds having both antimicrobial and 


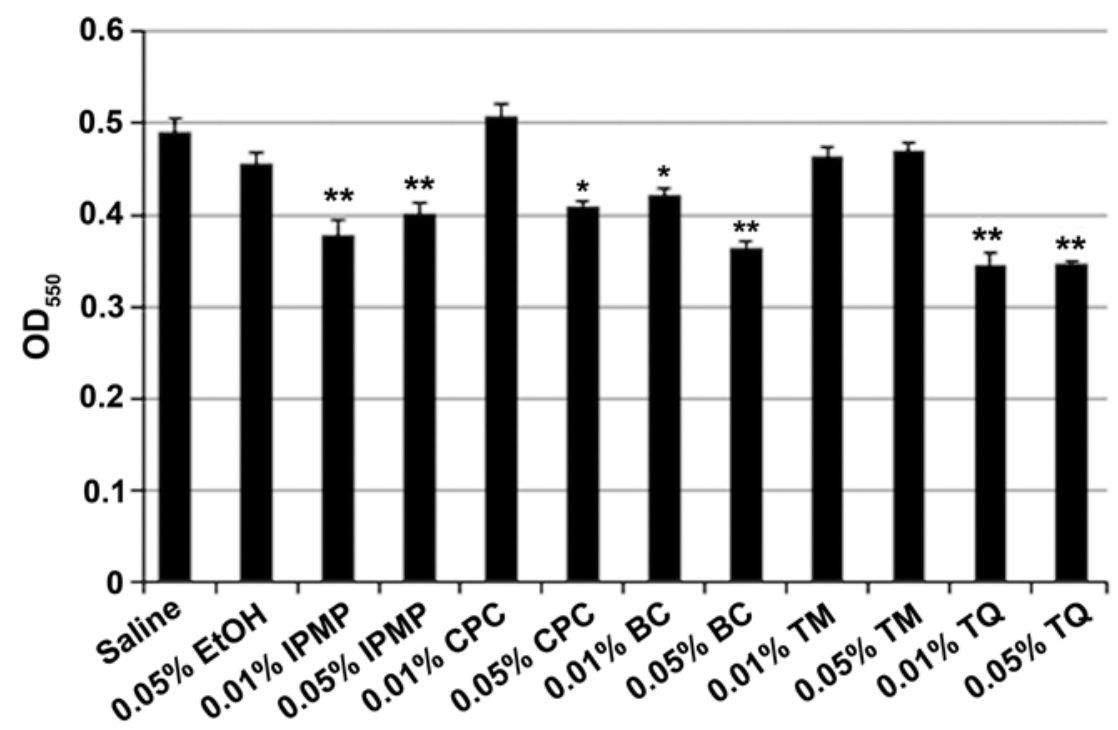

Figure 4. Biofilm assay with crystal violet after washing with indicated test reagents. Data are expressed as mean \pm standard deviation from three repeats. Differences were analyzed by ANOVA followed by Dunnett's test. ${ }^{*} \mathrm{P}<0.05$ or ${ }^{* *} \mathrm{P}<0.01$ vs. Saline.

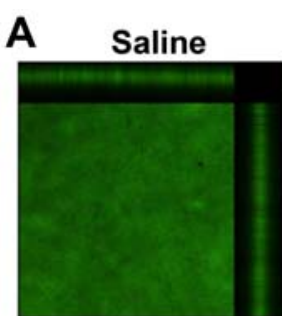

$0.01 \% \mathrm{TQ}$

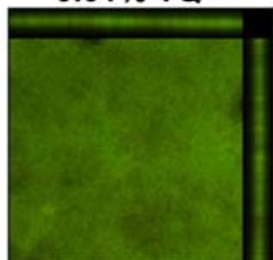

$0.01 \%$ IPMP

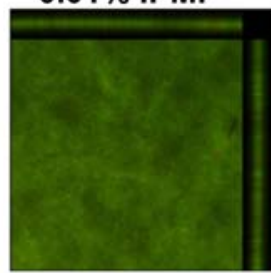

$0.01 \%$ BC

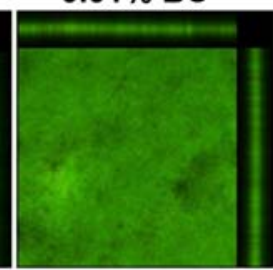

$0.01 \%$ TM

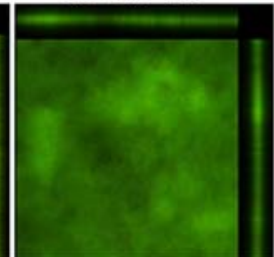

$0.01 \%$ CPC
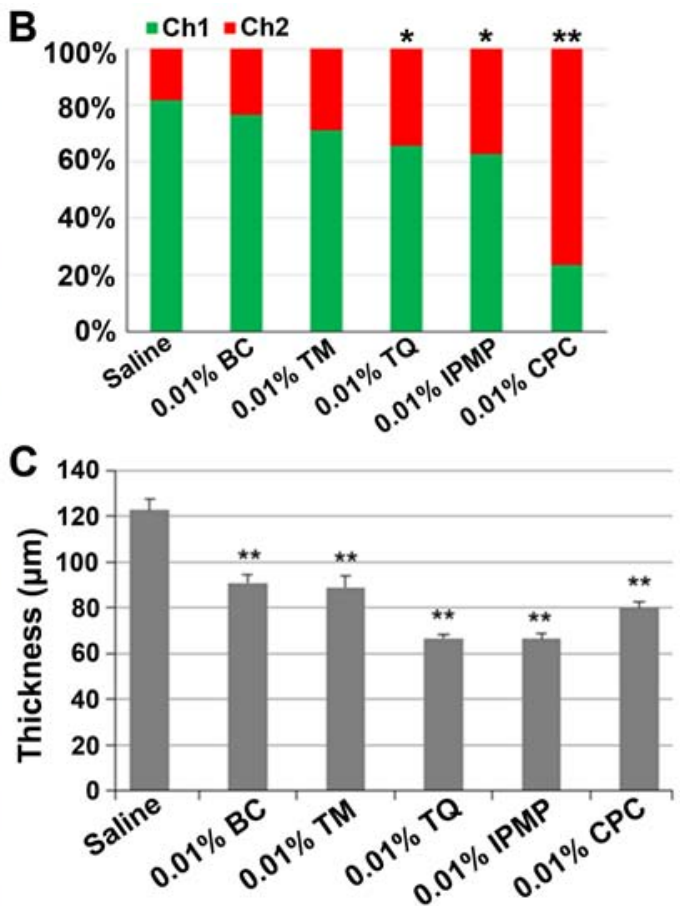

Figure 5. Confocal laser scanning microscopy analysis of dual species biofilms of FN and AN after treatment with indicated test reagents. (A) Merged images of biofilms stained with SYTO-9 (green) and propidium iodide (PI, red), which indicate live and dead cells, respectively. (B) Arithmetic average luminance of SYTO-9 (Ch1) and PI (Ch2) in the tested biofilm. Difference was analyzed by Chi-squared test. ${ }^{*} \mathrm{P}<0.05$ or ${ }^{* *} \mathrm{P}<0.01$ vs. Saline. (C) Average thickness of biofilm retained after treatment. Data are expressed as mean \pm standard deviation from three repeats. Differences were analyzed by ANOVA followed by Dunnett's test. ${ }^{* *} \mathrm{P}<0.01$ vs. Saline. FN, Fusobacterium nucleatum; AN, Actinomyces naeslundii; TM, thymol; TQ, thymoquinone; IPMP, isopropyl methylphenol; BC, black cumin; CPC, cetylpyridinium chloride.

anti-inflammatory activity are ideal therapeutics for periodontal diseases. TQ, a main component of BC essential oil, exhibits antimicrobial activity as well as anti-inflammatory effects (26-29). In this study we examined the potential of this herbal quinone as a therapeutic to treat $\mathrm{FN}$-associated periodontitis. To the best of our knowledge, this is the first report demonstrating that TQ shows suppressive effects on $\mathrm{FN}$-associated biofilm and inflammation.
Although we attempted to prepare FN biofilm in multi-titer plates to examine the antibiofilm effect of TQ, FN did not form stable biofilm. We hypothesized that FN requires cooperation from other members of the oral microbiota to establish intimate adherence to material surfaces. To test this hypothesis, we co-cultivated FN with well-known early dental colonizers, AN or SM. Co-cultivation of FN and AN significantly increased biofilm mass, and microscopic analysis of biofilm suspensions 


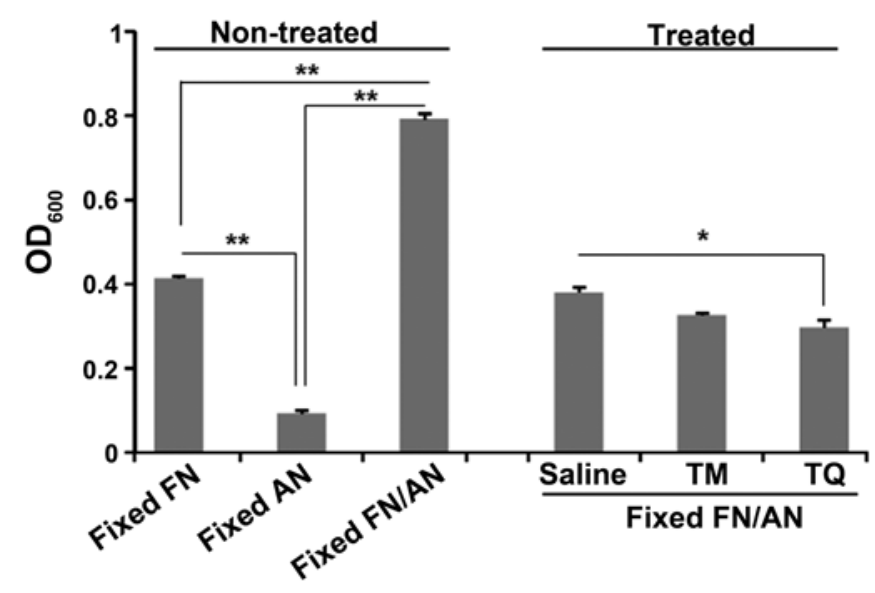

Figure 6. Cleansing effect of TM and TQ on prefixed FN/AN biofilm. After FN and AN were fixed with 4\% PFA, a dual species biofilm was prepared by incubating the fixed bacteria for $24 \mathrm{~h}$. The biofilm mass of untreated or samples washed with saline, TM $(0.01 \%)$ or TQ $(0.01 \%)$ was compared. Statistical differences among the samples in non-treated and treated experiments were separately evaluated ANOVA followed by Turkey's test. Significant difference is indicated by ${ }^{* *} \mathrm{P}<0.01$ or ${ }^{*} \mathrm{P}<0.05$ vs. samples indicated by lines. TM, thymol; TQ, thymoquinone; FN, Fusobacterium nucleatum; AN, Actinomyces naeslundii; PFA, paraformaldehyde.
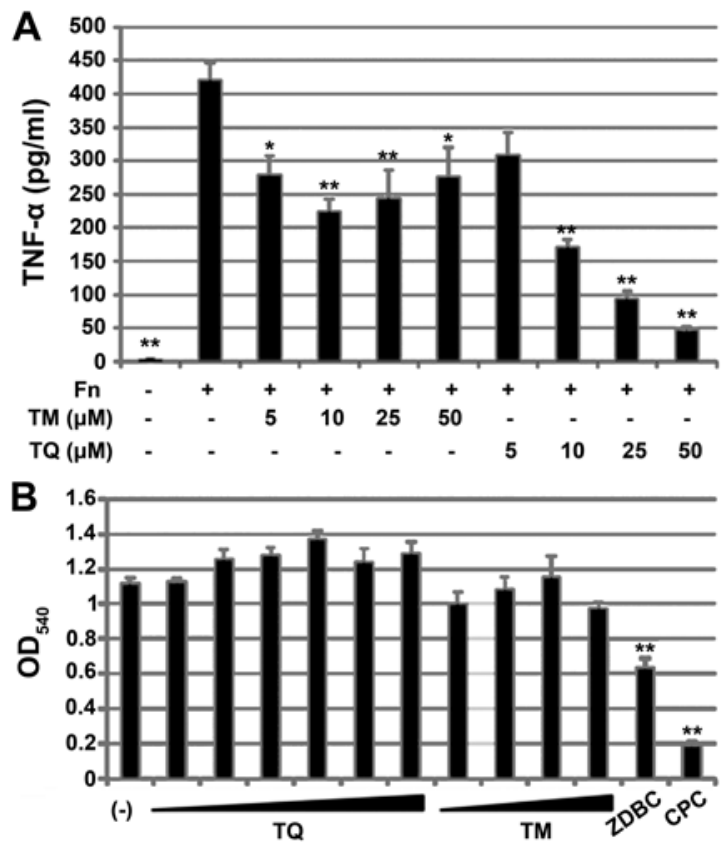

Figure 7. Effect of TQ and TM on FN-mediated proinflammatory response in the human monocytic cell line THP-1. (A) TNF- $\alpha$ secreted from THP-1 after stimulation of FN strain ATCC25586 (Fn). THP-1 pretreatment before Fn-stimulation is indicated below the panel. Data are expressed as mean \pm standard deviation from three repeats. Differences were analyzed by ANOVA followed by Dunnett's test. Significant difference from FN-stimulation alone is shown by ${ }^{*} \mathrm{P}<0.05$ or ${ }^{* *} \mathrm{P}<0.01$. (B) MTT assay of THP-1 with or without TQ treatment $(1,2.5,5,10,25$ or $50 \mu \mathrm{M})$, TM $(5,10$, 25 or $50 \mu \mathrm{M}), 1 \mathrm{mg} / \mathrm{ml} \mathrm{ZDBC}$ or $50 \mu \mathrm{M}$ CPC under FN-stimulation. Data are expressed as mean \pm standard deviation of three repeats. Differences were analyzed by ANOVA followed by Dunnett's test. Significant differences from FN-stimulation alone (indicated by -) are indicated by ${ }^{* *} \mathrm{P}<0.01$. TQ, thymoquinone; TM, thymol; FN, Fusobacterium nucleatum; TNF, tumor necrosis factor; CPC, cetylpyridinium chloride; ZDBC, zinc dibutyldithiocarbamate.

showed co-aggregation of these species. Multispecies biofilms having FN/AN as the core components could serve as an in vitro subgingival plaque model. Here, increases in FN/AN biofilm mass appeared to be mainly attributable to FN since inactivation of FN with TQ pretreatment abolished this increase (Fig. 3).

Microbial biofilm typically contains extracellular polysaccharides (3), but SEM examination of FN/AN-mixed biofilms showed no mucous matrices within co-aggregates. Although aggregation factors that might mediate FN/AN binding await elucidation, these interactions likely involve physicochemical interactions of surface molecules, since FN/AN co-aggregation was observed even after fixation with PFA. Interestingly, co-cultivation of FN and SM reduced biofilm mass over that seen for cultures containing FN alone. In addition, SM inhibited FN/AN biofilm formation. This result suggests that some resident Streptococci in the oral environment could have inhibitory effects on FN-associated biofilms by competing with binding receptors. Use of broad-spectrum antimicrobials for oral hygiene care can compromise such indigenous anti-FN biofilm activity of oral microbiome. Natural compounds such as herbal extracts have mild and diverse pharmacological actions, and thus are becoming attractive targets to develop oral hygiene care products that have lower toxicity toward both beneficial components of the oral microbiota as well as to human cells (15).

The BC essential oil components TM and TQ significantly inhibited formation of FN/AN dual species biofilms. This effect was due to growth inhibition of both species because the optical density of the culture was not increased during incubation. The bactericidal effect of TM or TQ appeared to be weak as evidenced by CLSM results showing that after treatment with $0.01 \% \mathrm{TM}$ or TQ over $70 \%$ of the bacteria within the biofilms survived whereas treatment with $0.01 \%$ CPC killed over $70 \%$ of the bacteria. Previous reports indicated that TQ has more pronounced antimicrobial activity against Gram-positive bacteria than Gram-negative bacteria $(26,27)$. However, in this study FN seemed to be more sensitive to TQ than AN since biofilm mass was reduced with TQ pretreatment of FN but not AN (Fig. 3). Thus, oral hygiene care using $\mathrm{BC}$ oil and its components might inhibit dental plaque formation by affecting a relatively narrow spectrum of bacteria and minimizing damage to the healthy oral microbiome.

The most notable finding in this study was that TQ reduced the mass of established FN/AN biofilms. This cleansing effect was equivalent to that for IPMP and greater than that seen for CPC. A similar trend was observed by CLSM, in which the thickness of FN/AN biofilms was decreased by treatment with TQ or IPMP to a greater degree than that seen for TM and CPC. TM and CPC treatment resulted in a significant reduction in FN/AN biofilm thickness, but did not decrease biofilm mass as evaluated by crystal violet staining (Figs. 4 and 5). These results might indicate that TQ and IPMP compromise FN/AN aggregation whereas TM and CPC condense biofilm structures.

The biofilm assay using prefixed FN and AN (Fig. 6) indicated that the cleansing effect of TQ on FN/AN biofilms likely involves interference with cell surface interactions. Since TQ is hydrophobic and localizes to the membrane fraction of BC seeds, TQ may be able to penetrate the outer membrane of bacteria to affect membrane integrity, which in turn can 
compromise FN/AN binding. In fact, TQ is reported to induce membrane damage in pathogenic fungi (30).

Subgingival inflammation is one of the pathologies of chronic periodontitis. Prolonged inflammation results in gingival tissue damage and alveolar bone destruction. FN lipopolysaccharides are known to induce extensive inflammatory responses mediated through TLR2 and TLR4 signaling (5). TQ is reported to repress inflammatory responses via diverse actions including inhibition of 5-lipoxygenase and leukotriene $\mathrm{C} 4$ synthase (26), inducible nitric oxide synthase (31), suppression of $\mathrm{NF}-\kappa \mathrm{B}$ signaling pathways (32) and enhanced production of antioxidants such as catalase or glutathione $(33,34)$. Consistent with these findings, here we showed that TQ repressed TNF- $\alpha$ production from a human monocytic cell line in response to FN (Fig. 7). This effect was not due to TQ cytotoxicity to THP-1 cells since cell viability was not affected at TQ concentrations of up to $50 \mathrm{uM}$, indicating that TQ does not compromise immunological clearance of oral pathogens. Surprisingly, CPC, which is widely used as an active component of oral care products, showed greater cytotoxicity to THP-1 cells than did ZDBC. Meanwhile, with an estimated $\mathrm{LD}_{50}$ of around $2.4 \mathrm{~g} / \mathrm{kg}$ (range 1.52-3.77), TQ is considered to have a low level of toxicity $(19,35)$ and only two cases of contact dermatitis have been reported in clinical studies involving BC oil $(36,37)$.

A limitation of this study is that all experiments were performed in vitro. Dental plaque is more complex and diverse among individuals and under in vivo conditions. Although a suppressive effect of TQ on alveolar bone loss and dental plaque accumulation has been demonstrated in a rat periodontitis model (22) and clinical study (38), respectively, these studies did not monitor microbiome alterations in saliva or dental plaque. Animal and clinical studies that focus on FN, a keystone periodontal pathogen, will provide new insights into the mechanisms for prophylactic and therapeutic effects of TQ on periodontal disease.

In conclusion, the results of this study showed that TQ effectively removes established FN/AN biofilms in vitro by compromising FN binding to other dental plaque colonizers. TQ effectively inactivated FN and suppressed inflammatory response to this periodontal pathogen. Based on the crucial role of $\mathrm{FN}$ in dental plaque maturation, agents that target $\mathrm{FN}$, such as TQ, are candidates for safe and effective active ingredients in oral hygiene care products.

\section{Acknowledgements}

The authors would like to thank Dr. Hidenobu Senpuku (National Institute of infectious Diseases in Japan) for the gift of Actinomyces neaslandii strain X600.

\section{Funding}

This work was supported by JSPS KAKENHI (grant no. $18 \mathrm{~K} 17028)$.

\section{Availability of data and materials}

All data generated or analyzed during this study are included in this published article.

\section{Authors' contributions}

AT, MI, KS and TK designed the current study. AT, HNI, HYag and ME performed experiments to evaluate the effect of TQ on FN-containing biofilm. TN performed statistical analyses. HYam performed electron microscopic analyses. MI, KS and TK wrote the manuscript.

\section{Ethics approval and consent to participate}

Not applicable.

\section{Patient consent for publication}

Not applicable.

\section{Competing interests}

The authors declare that they have no competing interests.

\section{References}

1. Dewhirst FE, Chen T, Izard J, Paster BJ, Tanner AC, Yu WH, Lakshmanan A and Wade WG: The human oral microbiome. J Bacteriol 192: 5002-5017, 2010

2. Kolenbrander PE and London J: Adhere today, here tomorrow: Oral bacterial adherence. J Bacteriol 175: 3247-3252, 1993.

3. Rickard AH, Gilbert P, High NJ, Kolenbrander PE and Handley PS: Bacterial coaggregation: An integral process in the development of multi-species biofilms. Trends Microbiol 11: 94-100, 2003.

4. Han YW: Fusobacterium nucleatum: A commensal-turned pathogen. Curr Opin Microbiol 23: 141-147, 2015.

5. Sun Y, Shu R, Li CL and Zhang MZ: Gram-negative periodontal bacteria induce the activation of Toll-like receptors 2 and 4, and cytokine production in human periodontal ligament cells. J Periodontol 81: 1488-1496, 2010.

6. To TT, Gümüş P, Nizam N, Buduneli $N$ and Darveau RP: Subgingival plaque in periodontal health antagonizes at Toll-like receptor 4 and inhibits E-selectin expression on endothelial cells. Infect Immun 84: 120-126, 2015.

7. Hong CY, Lin SK, Kok SH, Cheng SJ, Lee MS, Wang TM, Chen CS, Lin LD and Wang JS: The role of lipopolysaccharide in infectious bone resorption of periapical lesion. J Oral Pathol Med 33: 162-169, 2004.

8. Suwabe K, Yoshida Y, Nagano K and Yoshimura F: Identification of an L-methionine $\gamma$-lyase involved in the production of hydrogen sulfide from L-cysteine in Fusobacterium nucleatum subsp. nucleatum ATCC 25586. Microbiology 157: 2992-3000, 2011.

9. Nakano M, Shin K, Wakabayashi H, Yamauchi K, Abe F and Hironaka S: Inactivating effects of the lactoperoxidase system on bacterial lyases involved in oral malodour production. J Med Microbiol 64: 1244-1252, 2015.

10. Rubinstein MR, Wang X, Liu W, Hao Y, Cai G and Han YW: Fusobacterium nucleatum promotes colorectal carcinogenesis by modulating E-cadherin/ $\beta$-catenin signaling via its FadA adhesin. Cell Host Microbe 14: 195-206, 2013.

11. Allen-Vercoe E, Strauss J and Chadee K: Fusobacterium nucleatum: An emerging gut pathogen? Gut Microbes 2: 294-298, 2011.

12. Seymour GJ, Ford PJ, Cullinan MP, Leishman S and Yamazaki K: Relationship between periodontal infections and systemic disease. Clin Microbiol Infect 13 (Suppl 4): S3-S10, 2007.

13. ImaiH,KitaF,IkesugiS,AbeM,SogabeS,Nishimura-DanjobaraY, Miura $\mathrm{H}$ and Oyama Y: Cetylpyridinium chloride at sublethal levels increases the susceptibility of rat thymic lymphocytes to oxidative stress. Chemosphere 170: 118-123, 2010.

14. Hidalgo E and Dominguez C: Mechanisms underlying chlorhexidine-induced cytotoxicity. Toxicol In Vitro 15: 271-276, 2001.

15. Chandra Shekar BR, Nagarajappa R, Suma S and Thakur R: Herbal extracts in oral health care-A review of the current scenario and its future needs. Pharmacogn Rev 9: 87-92, 2015. 
16. Darvishi Khezri H, Haidari Gorji MA, Morad A and Gorji H: Comparison of the antibacterial effects of matrica \& Persica ${ }^{\mathrm{TM}}$ and chlorhexidine gluconate mouthwashes in mechanically ventilated ICU patients: A double blind randomized clinical trial Rev Chilena Infectol 30: 361-373, 2013 (In Spanish)

17. Pedrazzi V, Leite MF, Tavares RC, Sato S, do Nascimento GC and Issa JP: Herbal mouthwash containing extracts of Baccharis dracunculifolia as agent for the control of biofilm: Clinical evaluation in humans. ScientificWorldJournal 2015: 712683, 2015.

18. Filoche SK, Soma K and Sissons CH: Antimicrobial effects of essential oils in combination with chlorhexidine digluconate. Oral Microbiol Immunol 20: 221-225, 2005.

19. Ali BH and Blunden G: Pharmacological and toxicological properties of Nigella sativa. Phytother Res 17: 299-305, 2003.

20. Gholamnezhad Z, Havakhah S and Boskabady MH: Preclinical and clinical effects of Nigella sativa and its constituent, thymoquinone: A review. J Ethnopharmacol 190: 372-386, 2016.

21. Kokoska L, Havlik J, Valterova I, Sovova H, Sajfrtova M and Jankovska I: Comparison of chemical composition and antibacterial activity of Nigella sativa seed essential oils obtained by different extraction methods. J Food Prot 71: 2475-2480, 2008.

22. Ozdemir H, Kara MI, Erciyas K, Ozer H and Ay S: Preventive effects of thymoquinone in a rat periodontitis model: A morphometric and histopathological study. J Periodontal Res 47: 74-80, 2012.

23. Nakasugi T, Murakawa T, Shibuya $\mathrm{K}$ and Morimoto $\mathrm{M}$ : Deodorizing substance in black cumin (Nigella sativa L.) seed oil. J Ole Sci 66: 877-882, 2017.

24. Saygun I, Nizam N, Keskiner I, Bal V, Kubar A, Acikel C, Serdar M and Slots J: Salivary infectious agents and periodontal disease status. J Periodontal Res 46: 235-239, 2011.

25. Zhou X, Liu X, Li J, Aprecio RM, Zhang W and Li Y: Real-time PCR quantification of six periodontal pathogens in saliva samples from healthy young adults. Clin Oral Investig 19: 937-946, 2015

26. Chaieb K, Kouidhi B, Jrah H, Mahdouani K and Bakhrouf A: Antibacterial activity of thymoquinone, an active principle of Nigella sativa and its potency to prevent bacterial biofilm formation. BMC Complement Altern Med 11: 29, 2011.

27. Kouidhi B,Zmantar T, Jrah H, Souiden Y, Chaieb K, Mahdouani K and Bakhrouf A: Antibacterial and resistance-modifying activities of thymoquinone against oral pathogens. Ann Clin Microbiol Antimicrob 10: 29, 2011.
28. Mansour M and Tornhamre S: Inhibition of 5-lipoxygenase and leukotriene $\mathrm{C} 4$ synthase in human blood cells by thymoquinone. J Enzyme Inhib Med Chem 19: 431-436, 2004

29. Woo CC, Kumar AP, Sethi G and Tan KH: Thymoquinone: Potential cure for inflammatory disorders and cancer. Biochem Pharmacol 83: 443-451, 2012.

30. Shokri H: A review on the inhibitory potential of Nigella sativa against pathogenic and toxigenic fungi. Avicenna J Phytomed 6: 21-33, 2016

31. El-Mahmoudy A, Matsuyama H, Borgan MA, Shimizu Y, El-Sayed MG, Minamoto $\mathrm{N}$ and Takewaki T: Thymoquinone suppresses expression of inducible nitric oxide synthase in rat macrophages. Int Immunopharmacol 2: 1603-1611, 2002.

32. Sethi G, Ahn KS and Aggarwal BB: Targeting nuclear factor-kappa B activation pathway by thymoquinone: Role in suppression of antiapoptotic gene products and enhancement of apoptosis. Mol Cancer Res 6: 1059-1070, 2008.

33. Mohamed A, Shoker A, Bendjelloul F, Mare A, Alzrigh M, Benghuzzi $\mathrm{H}$ and Desin T: Improvement of experimental allergic encephalomyelitis (EAE) by thymoquinone; an oxidative stress inhibitor. Biomed Sci Instrum 39: 440-445, 2003.

34. Sayed-Ahmed MM and Nagi MN: Thymoquinone supplementation prevents the development of gentamicin-induced acute renal toxicity in rats. Clin Exp Pharmacol Physiol 34: 399-405, 2007.

35. Badary OA, Al-Shabanah OA, Nagi MN, Al-Bekairi AM and Elmazar MMA: Acute and subchronic toxicity of thymoquinone in mice. Drug Develop Res 44: 56-61, 1998.

36. Steinmann A, Scatze M, Agathos M and Brett R: Allergic contact dermatitis from black cumin (Nigella sativa) oil after topical use. Contact Dermatitis 36: 268-269, 1997.

37. Zedlitz S, Kaufmann R and Bochncke WH: Allergic contact dermatitis from black cumin (Nigella sativa) oil-containing ointment. Contact Dermatitis 46: 188, 2002.

38. Kapil H, Suresh DK, Bathia SC and Arora KS: Assessment of clinical efficacy of locally delivered $0.2 \%$ thymoquinone gel in the treatment of periodontitis. Saudi Dent J 30: 348-354, 2018.

This work is licensed under a Creative Commons Attribution-NonCommercial-NoDerivatives 4.0 International (CC BY-NC-ND 4.0) License. 